\title{
Experimental Verification of Predicted Beam-Polarization Oscillations near a Spin Resonance
}

\author{
V. S. Morozov, A. W. Chao, ${ }^{*}$ A. D. Krisch, M. A. Leonova, R. S. Raymond, D. W. Sivers, and V. K. Wong \\ Spin Physics Center, University of Michigan, Ann Arbor, Michigan 48109-1040, USA
}

A. Garishvili, R. Gebel, A. Lehrach, B. Lorentz, R. Maier, D. Prasuhn, H. Stockhorst, and D. Welsch

Forschungszentrum Jülich, Institut für Kernphysik, Postfach 1913, D-52425 Jülich, Germany

F. Hinterberger and K. Ulbrich

Helmholtz-Institut für Strahlen- und Kernphysik, Universität Bonn, D-53115 Bonn, Germany

A. Schnase

JAEA/J-PARC, Ring RF Group, Shirakata-Shirane 2-4, Tokai-Mura, Ibaraki 319-1195, Japan

E. J. Stephenson

IUCF, Indiana University, Bloomington, Indiana 47408-0768 USA

N.P. M. Brantjes, C. J. G. Onderwater, and M. da Silva

University of Groningen, Groningen, The Netherlands

(Received 22 September 2007; published 5 February 2008)

\begin{abstract}
The Chao matrix formalism allows analytic calculations of a beam's polarization behavior inside a spin resonance. We recently tested its prediction of polarization oscillations occurring in a stored beam of polarized particles near a spin resonance. Using a $1.85 \mathrm{GeV} / c$ polarized deuteron beam stored in the COoler SYnchrotron, we swept a new rf solenoid's frequency rather rapidly through $400 \mathrm{~Hz}$ during $100 \mathrm{~ms}$, while varying the distance between the sweep's end frequency and the central frequency of an rf-induced spin resonance. Our measurements of the deuteron's polarization near and inside the resonance agree with the Chao formalism's predicted oscillations.
\end{abstract}

DOI: 10.1103/PhysRevLett.100.054801

PACS numbers: 29.27.Bd, 29.27.Hj, 41.75.Ak

Polarized stored hadron and lepton beams often provide the best technique for studying the spin dependence of hadronic interactions in the $1 \mathrm{GeV} / c$ to $1 \mathrm{TeV} / c$ region. Polarized beam experiments at storage rings [1-5] need the ability to precisely control the beam's polarization. A stored beam's polarization can be manipulated in a wellcontrolled way by ramping an rf magnet's frequency through an rf-induced spin resonance.

The Froissart-Stora (F-S) formula [6] has been widely used to calculate a beam's polarization after crossing a spin resonance. However, it is valid only for a constant-rate linear crossing from far below to far above the resonance. Chao's new matrix formalism was proposed [7] to deal with conditions where the F-S formula is not valid; the Chao formalism can be used to calculate the spin dynamics anywhere inside a piecewise linear resonance crossing. An earlier experiment [8] at the COoler SYnchrotron (COSY) first tested the Chao formalism's predicted spin behavior by sweeping an rf dipole's frequency near or through an rfinduced spin resonance. That experiment suggested that a faster crossing rate was needed to test its striking prediction of large polarization oscillations near the resonance. This Letter describes a faster crossing rate experiment at COSY.

In an ideal flat circular storage ring or accelerator, with no horizontal magnetic fields, each particle's spin pre- cesses around the vertical magnetic fields of the ring's bending dipoles. The spin tune $\nu_{s}$, which is the number of spin precessions during one turn around the ring, is proportional to the particle's energy; $\nu_{s}=G \gamma$, where $G=(g-2) / 2$ is its gyromagnetic anomaly and $\gamma$ is its Lorentz energy factor. Horizontal rf magnetic fields can induce an rf spin resonance $[6,9,10]$, which can be used to spin-manipulate the stored particles [11-16]. For deuterons, the rf-induced spin resonance's frequency is

$$
f_{r}=f_{c}\left(k \pm G_{d} \gamma\right)
$$

where $f_{c}$ is the deuteron's circulation frequency, $k$ is an integer, and $G_{d}=-0.142987$.

Ramping an rf magnet's frequency through a spin resonance with resonance strength $\mathcal{E}$ can rotate a stored beam's polarization. When its frequency is ramped at a constant rate, during a ramp time $\Delta t$, by a range $\Delta f$, from far below to far above a resonance, the Froissart-Stora equation [6] can relate the beam's initial vector polarization $P_{i}$ and its polarization $P_{f}$ after crossing the resonance,

$$
P_{f}=P_{i}\left\{2 \exp \left[-\frac{\left(\pi \mathcal{E} f_{c}\right)^{2}}{\Delta f / \Delta t}\right]-1\right\}
$$

As discussed earlier [8], Chao's matrix formalism [7] for spin dynamics allows one to analytically solve the spin equation of motion near an isolated spin resonance, if its 
crossing can be expressed as a series of linear segments. Each segment must have a fixed or linearly changing distance between the spin tune $\nu_{s}=G \gamma$ and the resonance tune $\nu_{r} \equiv k \pm f_{r} / f_{c}$. After obtaining, for each segment, the time-dependent matrix describing a spinor's evolution in the segment, one multiplies these matrices sequentially to obtain the final polarization $P_{f}$.

We recently tested Chao's matrix formalism with the technique shown in Fig. 1 using our new rf solenoid; it is a 25-turn air-core water-cooled copper coil, of length $57.5 \mathrm{~cm}$ and average diameter $21 \mathrm{~cm}$. Its inductance was $41 \pm 3 \mu \mathrm{H}$, and its longitudinal rf magnetic field was about $1.25 \mathrm{mT}$ at its center. It was part of an RLC resonant circuit, which operated near $917 \mathrm{kHz}$, typically at an $\mathrm{rf}$ voltage of $5.7 \mathrm{kV} \mathrm{rms} \mathrm{producing} \mathrm{a} \mathrm{longitudinal} \mathrm{rf} \int B_{\mathrm{rms}} d l$ of $0.69 \pm 0.05 \mathrm{~T} \mathrm{~mm}$.

The other apparatus for this experiment, including the COSY storage ring [17-20], the EDDA polarimeter [21,22], the electron cooler [23], the low energy polarimeter [24], the injector cyclotron, and the polarized ion source [25-27] were shown in Fig. 4 of Ref. [8]. The beam from the polarized $D^{-}$ion source was accelerated by the cyclotron to $75.7 \mathrm{MeV}$ and then strip-injected into COSY. The Low Energy Polarimeter measured the $D^{-}$beam's polarization before injection into COSY to monitor the cyclotron's and the ion source's stability.

The EDDA polarimeter [21,22] measured the beam's polarization in COSY. We reduced its systematic errors by repeatedly cycling the beam produced by the polarized deuteron ion source through five different vector $P_{V}$ and tensor $P_{T}$ vertical polarization states:

$$
\left(P_{V}, P_{T}\right)=(0,0),(+1,+1),\left(-\frac{1}{3},-1\right),\left(-\frac{2}{3}, 0\right),(-1,+1) .
$$

The asymmetry measured in the $(0,0)$ spin state was subtracted from the other measured asymmetries, in each $20 \mathrm{~ms}$ time bin, to correct for detector efficiencies and beam motion asymmetries in the EDDA polarimeter.

In COSY, the deuterons' average circulation frequency $f_{c}$ was $1.14743 \mathrm{MHz}$ at $1.85 \mathrm{GeV} / c$, where their Lorentz energy factor was $\gamma=1.4046$. For these parameters, the

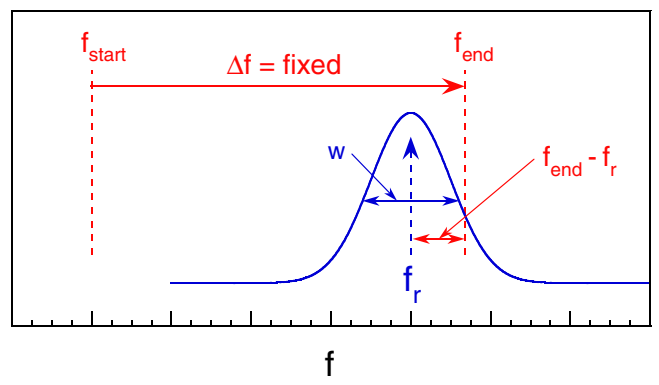

FIG. 1 (color online). Schematic of the Chao formalism test. The rf solenoid's frequency $f_{\text {rf }}$ was ramped, by a fixed range $\Delta f$, for different distances between the ramp's end frequency $f_{\text {end }}$ and the resonance's center $f_{r}$. The curve shows the resonance with a total (FWHM) width $w$. spin tune $\nu_{s}=G_{d} \gamma$ was -0.20084 . Thus, Eq. (1) implies that the $k=1$ spin resonance's central frequency should be very near $f_{r}=\left(1+G_{d} \gamma\right) f_{c}=917.0 \mathrm{kHz}$.

We measured the rf solenoid's strength $\mathcal{E}$ by measuring the polarization after ramping its frequency through the resonance with various ramp times $\Delta t$ with its frequency range $\Delta f$ and voltage fixed, as shown in Fig. 2. We then fit these data to Eq. (2), the Froissart-Stora equation [6], to obtain the measured value of $\mathcal{E}$.

To study the Chao formalism's predicted dependence on the beam's momentum spread $\Delta p / p$, we varied the $20.6 \mathrm{keV}$ electron Cooler's on-time at injection. It cooled the deuterons' emittances both longitudinally and transversely for 15 or $25 \mathrm{~s}$. The deuterons were then accelerated to $1.85 \mathrm{GeV} / c$. The rf acceleration cavity was off and shorted during COSY's flat top; thus, there were no synchrotron oscillations.

We tested the Chao formalism by ramping the rf solenoid's frequency over a range $\Delta f$, which started at $f_{\text {start }}$ (well outside the rf spin resonance centered at $f_{r}$ ) and ended at $f_{\text {end }}$ near or inside the resonance, as shown in Fig. 1. For each $f_{\text {end }}$ data point, both $\Delta f$ and the ramp time $\Delta t$ were held fixed, at $400 \mathrm{~Hz}$ and $100 \mathrm{~ms}$, respectively, while $f_{\text {end }}$ was set to the values shown in Figs. 3 and 4. After $f_{\text {rf }}$ reached $f_{\text {end }}$, the rf solenoid was turned off abruptly (in a few $\mu$ s) to preserve the vertical polarization at that instant. We then measured the deuterons' vector asymmetry in all five $\left(P_{V}, P_{T}\right)$ states. The resulting final vector polarization $P_{f}$ for each nonzero spin state is plotted vs $f_{\text {end }}$ in Figs. 3 and 4.

We first calculated the Chao formalism's prediction for $\Delta p / p=0$ by inserting, into Eqs. (4)-(9) of Ref. [8], our measured $\mathcal{E}=1.06 \times 10^{-5}$ from Fig. 2 , our $\Delta f$ of $400 \mathrm{~Hz}$, and our $\Delta t$ of $100 \mathrm{~ms}$. To take into account the beam's

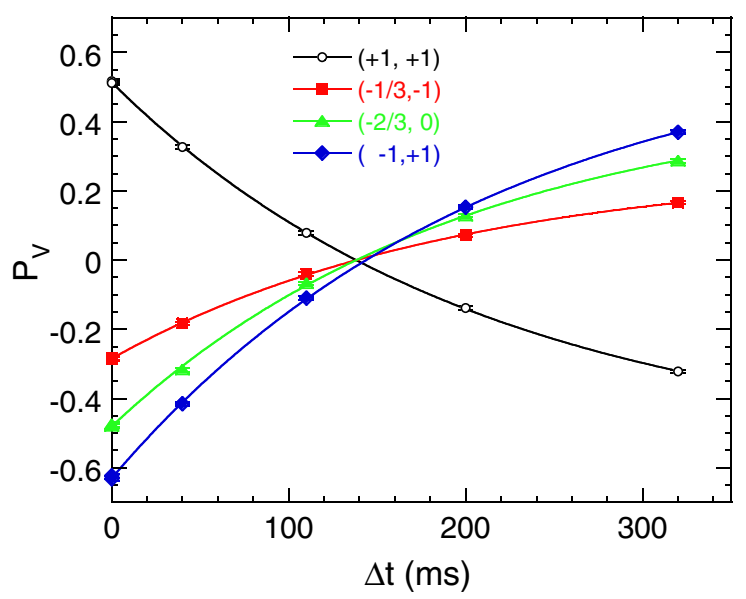

FIG. 2 (color online). Measured $1.85 \mathrm{GeV} / c$ deuteron vector polarizations plotted vs rf-solenoid ramp time $\Delta t$ with $15 \mathrm{~s}$ electron cooling for the four indicated spin states. Their averaged fit to Eq. (2) gave $\mathcal{E}$ of $(1.05 \pm 0.01) \times 10^{-5}$. The above $\Delta t$ curve's frequency range $\Delta f$ was $300 \mathrm{~Hz}$. Averaging 13 similar $\Delta t$ curves gave $\mathcal{E}$ of $(1.060 \pm 0.005) \times 10^{-5}$. 


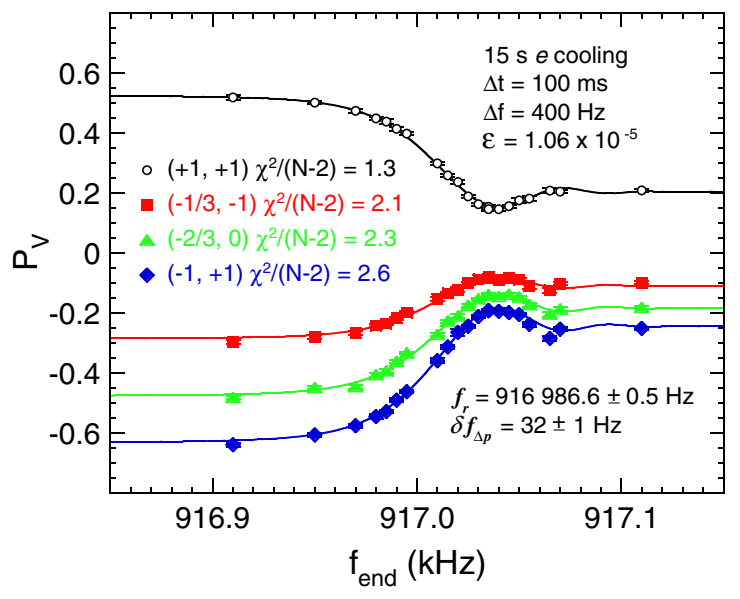

FIG. 3 (color online). Measured $1.85 \mathrm{GeV} / c$ deuteron vector polarizations plotted vs rf-solenoid end frequency $f_{\text {end }}$. Its ramp time $\Delta t$ was $100 \mathrm{~ms}$; its frequency range $\Delta f$ was $400 \mathrm{~Hz}$, and its $\mathcal{E}$ was $1.06 \times 10^{-5}$. The Chao formalism fits, shown by lines, gave a resonance frequency $f_{r}$ of $916986.6 \pm 0.5 \mathrm{~Hz}$ and a Gaussian $\delta f_{\Delta p}$ of $32 \pm 1 \mathrm{~Hz}$ FWHM. Only the statistical errors were used to calculate $\chi^{2} /(N-2)$; $e$ cooling was on for $15 \mathrm{~s}$.

momentum spread $\Delta p / p$, we next folded this result together with Gaussians representing different values of the beam's $f_{r}$ spread, $\delta f_{\Delta p} \equiv \frac{d f_{r}}{d p} \Delta p$, due to $\Delta p / p$. We then fit the data in Figs. 3 and 4 with $f_{r}$ and $\delta f_{\Delta p}$ as the two free parameters. The Chao formalism fits are shown as solid lines for each nonzero spin state in each figure.

We calculated $\chi^{2} /(N-2)$ for each fit to compare its agreement with the data for each of the four nonzero spin states. Each $\chi^{2}$ analysis included only the data's statistical errors and ignored systematic errors; nevertheless, all $\chi^{2} /(N-2)$ were near 1 despite the curves' complex shapes. The oscillations' positions and magnitudes are very sensitive to the values of $f_{r}$ and $\delta f_{\Delta p}$, respectively.

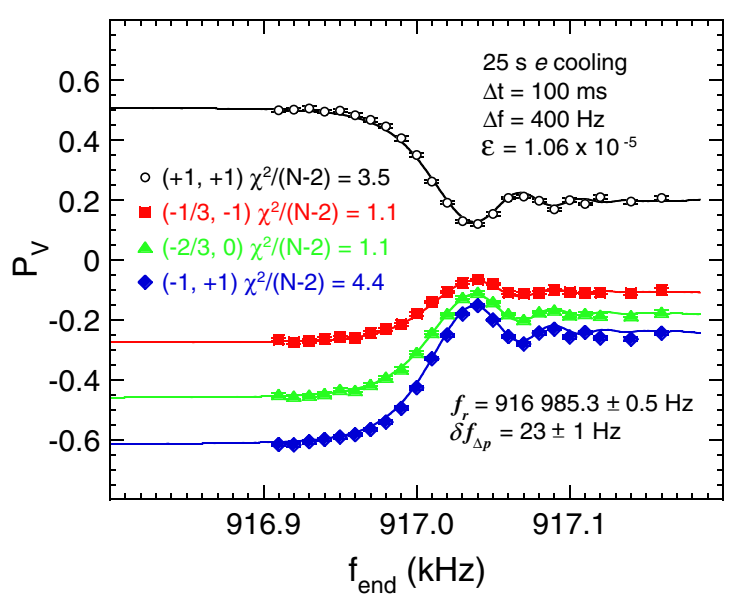

FIG. 4 (color online). Measured deuteron vector polarizations plotted vs $f_{\text {end }}$ as in Fig. 3. With $25 \mathrm{~s} e$ cooling, the fit curves gave $f_{r}$ of $916985.3 \pm 0.5 \mathrm{~Hz}$ and $\delta f_{\Delta p}$ of $23 \pm 1 \mathrm{~Hz}$ FWHM.
Minimizing the $\chi^{2} /(N-2)$ values in Fig. 3 gave $f_{r}$ of $916986.6 \pm 0.5 \mathrm{~Hz}$ and $\delta f_{\Delta p}$ of $32 \pm 1 \mathrm{~Hz}$ for $15 \mathrm{~s}$ electron cooling. Minimizing $\chi^{2} /(N-2)$ for the $25 \mathrm{~s}$ electron cooling data in Fig. 4 gave an $f_{r}$ of $916985.3 \pm 0.5 \mathrm{~Hz}$ and $\delta f_{\Delta p}$ of $23 \pm 1 \mathrm{~Hz}$. The $\chi^{2} /(N-2)$ analyses in Figs. 3 and 4 both support the Chao formalism [7,8]. As predicted, the oscillation amplitude increased as $\delta f_{\Delta p}$ decreased.

Figure 5 compares the $25 \mathrm{~s} e$-cooling data, shown in Fig. 4, with the prediction $[7,8]$ for $\mathcal{E}=1 \times 10^{-5}$. The fit with the measured $\mathcal{E}=(1.060 \pm 0.005) \times 10^{-5}$ agrees with the data in Fig. 5 far better than the prediction. This large difference clearly shows the strong sensitivity of such $\Delta f$ sweeps to changes in $\mathcal{E}$.

For each cooling time, we also measured the final polarization $P_{f}$ after the rf solenoid was run at many different fixed frequencies to independently determine the resonance's center $f_{r}$ and its FWHM-width $w$. These data are plotted vs the rf frequency $f_{\text {rf }}$ in Fig. 6, along with their fit values of $f_{r}$ and $w$ for the 15 and $25 \mathrm{~s}$ cooling times. The fit widths of $41 \pm 1$ and $29 \pm 1 \mathrm{~Hz}$ for the 15 and $25 \mathrm{~s}$ cooling times, respectively, should be equal to the folding together of the resonance's natural width $2 \mathcal{E} f_{c}=24 \mathrm{~Hz}$ and the $f_{r}$ spreads, $\delta f_{\Delta p}$, obtained by fitting the data in Figs. 3 and 4. Adding this $24 \mathrm{~Hz}$ width in quadrature with the 32 and $23 \mathrm{~Hz} \delta f_{\Delta p}$ values gives $40 \pm 2$ and $33 \pm 2 \mathrm{~Hz}$; these seem to be consistent with the $41 \pm 1$ and $29 \pm 1 \mathrm{~Hz}$ from Fig. 6, for cooling times of 15 and $25 \mathrm{~s}$, respectively.

For $25 \mathrm{~s}$ cooling, the $f_{r}$ of $916985.3 \pm 0.3 \mathrm{~Hz}$ from Fig. 4 and the $f_{r}$ of $916990 \pm 10 \mathrm{~Hz}$ from Fig. 6(b) differ by only $5 \mathrm{~Hz}$. [The Fig. 4 and 6(b) data were obtained sequentially.] But, for $15 \mathrm{~s}$ cooling, with 102 hours between the Fig. 3 and 6(a) data runs, the $f_{r}$ values are

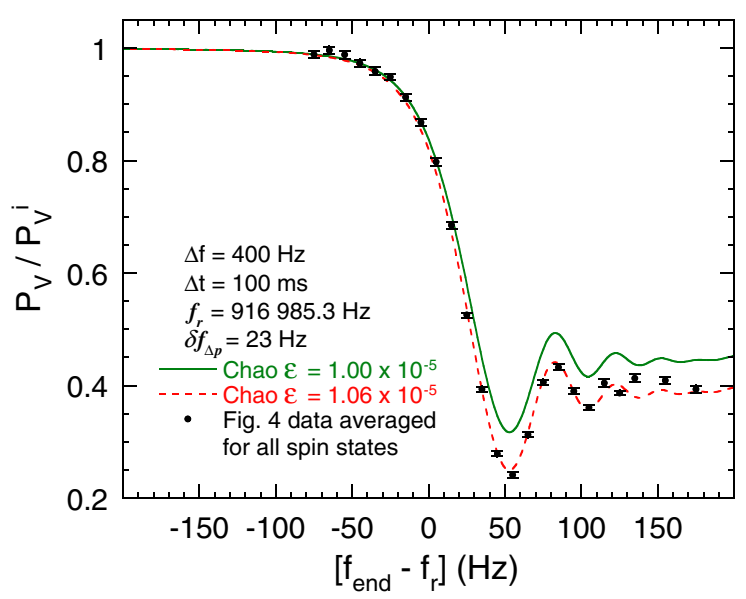

FIG. 5 (color online). Measured deuteron vector polarization ratios from Fig. 4, averaged for all four spin states, are plotted vs $f_{\text {end }}-f_{r}$. With $25 \mathrm{~s} e$ cooling, $f_{r}$ was $916985.3 \pm 0.5 \mathrm{~Hz}$ and $\delta f_{\Delta p}$ was $23 \pm 1 \mathrm{~Hz}$ FWHM. The solid line shows the prediction for $\mathcal{E}$ of $1.00 \times 10^{-5}$ found in Fig. 9 of Ref. [8]. The dashed line shows the fit, from Fig. 4, for the solenoid's measured resonance strength $\mathcal{E}$ of $1.060 \pm 0.005 \times 10^{-5}$. 


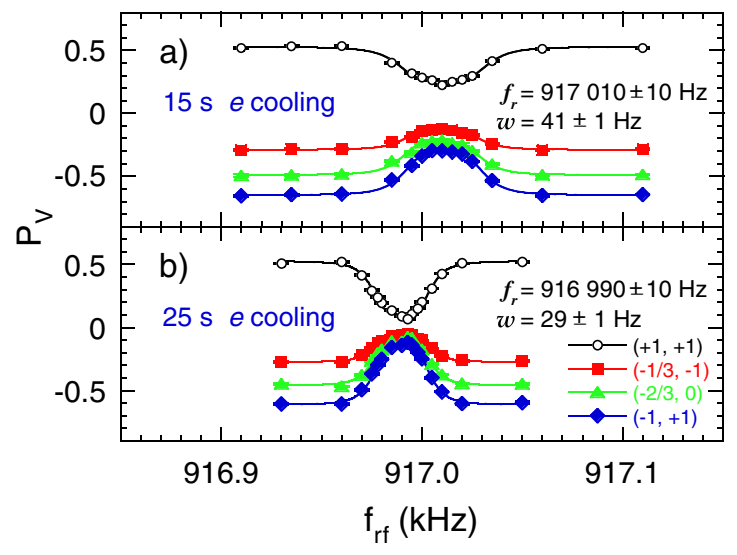

FIG. 6 (color online). Measured deuteron vector polarizations at $1.85 \mathrm{GeV} / c$ plotted vs the rf-solenoid fixed frequency $f_{\text {rf }}$. Fits to 2nd-order Lorentzians give (a) with $15 \mathrm{~s} e$ cooling, $f_{r}=917010 \pm 10 \mathrm{~Hz}$ and $w=41 \pm 1 \mathrm{~Hz}$ FWHM; (b) $f_{r}=916990 \pm 10 \mathrm{~Hz}$ and $w=29 \pm 1 \mathrm{~Hz}$ FWHM with $25 \mathrm{~s}$ cooling. The data points' errors are purely statistical; the errors in $f_{r}$ and $w$ include estimates of systematic errors due to COSY's stability at the few $\mathrm{Hz}$ level.

$916986.6 \pm 0.3 \mathrm{~Hz}$ and $917010 \pm 10 \mathrm{~Hz}$, respectively. This $23 \mathrm{~Hz}$ shift may be due to COSY's long-term stability.

In summary, we tested the Chao formalism's prediction of polarization oscillations when crossing an isolated spin resonance, in a region where the Froissart-Stora formula is not valid. Using $1.85 \mathrm{GeV} / c$ vertically polarized deuterons stored in COSY, we ramped an rf solenoid's frequency through a range $\Delta f$ ending near a spin resonance; the magnitudes of both $\Delta f$ and the ramp time $\Delta t$ were fixed, while we varied the ramp's distance from the resonance. The good fits to the precise data from our new type of experiment, shown in Figs. 3-5, clearly demonstrate the Chao formalism's $[7,8]$ ability to obtain the values of $f_{r}$, $\delta f_{\Delta p}$, and $\mathcal{E}$ from the measured polarization's behavior inside a spin resonance.

We thank the COSY staff for the successful operation of COSY with its injector cyclotron and polarized ion source. We are grateful to E.D. Courant, Ya.S. Derbenev, D. Eversheim, G. Fidecaro, W. Haeberli, H. Huang, A. M. Kondratenko, Yu.F. Orlov, H. Rohdjeß, T. Roser, H. Sato, W. Scobel, K. Yonehara, and others for their help and advice. This research was supported by grants from the German BMBF Science Ministry, the Netherlands NWO, and the U.S. NSF.

*Also at SLAC, 2575 Sand Hill Rd., Menlo Park, CA 94025.

[1] R. Alarcon et al. (BLAST Collaboration), MIT Bates Report No. 2-39, 1999.

[2] H. Rohdjess et al., in Results from EDDA@COSY: Spin Observables in Proton-Proton Elastic Scattering, edited by Y.I. Makdisi, W. W. MacKay, and A. U. Luccio, AIP Conf. Proc. No. 675 (AIP, Melville, NY, 2003), p. 523.

[3] Y. Makdisi, in Spin at RHIC, edited by K. J. Heller and S. L. Smith, AIP Conf. Proc. No. 343 (AIP, Woodbury, NY, 1995), p. 75.

[4] A. D. Krisch et al. (SPIN Collaboration), University of Michigan Report No. UM-HE 96-20, 1996; University of Michigan Report No. UM-HE 99-05, 1999.

[5] A. Airapetian et al. (HERMES Collaboration), DESYPRC Report No. 99-08, 1999.

[6] M. Froissart and R. Stora, Nucl. Instrum. Methods 7, 297 (1960).

[7] A.W. Chao, Phys. Rev. ST Accel. Beams 8, 104001 (2005).

[8] V.S. Morozov et al., Phys. Rev. ST Accel. Beams 10, 041001 (2007).

[9] E. D. Courant, Bull. Am. Phys. Soc. 7, 33 (1962); Report No. BNL-EDC-45, 1962.

[10] B. W. Montague, Phys. Rep. 113, 35 (1984).

[11] V. S. Morozov et al., Phys. Rev. Lett. 91, 214801 (2003).

[12] K. Yonehara et al., in Spin-Flipping Polarized Deuterons at COSY, edited by Z. Parsa, AIP Conf. Proc. No. 698 (AIP, Melville, NY, 2003), p. 763.

[13] M. A. Leonova et al., Phys. Rev. Lett. 93, 224801 (2004).

[14] V.S. Morozov et al., Phys. Rev. ST Accel. Beams 8, 061001 (2005).

[15] M. A. Leonova et al., Phys. Rev. ST Accel. Beams 9, 051001 (2006).

[16] A.D. Krisch et al., Phys. Rev. ST Accel. Beams 10, 071001 (2007).

[17] R. Maier, Nucl. Instrum. Methods Phys. Res., Sect. A 390, 1 (1997).

[18] A. Lehrach et al., in Proceedings of the 1999 Particle Accelerator Conference, New York, 1999, edited by A. Luccio and W. MacKay (IEEE, Piscataway, NJ, 1999), p. 2292.

[19] H. Stockhorst et al., in Proceedings of the 8th European Particle Accelerator Conference, Paris, 2002 (EPS-IGA/ CERN, Geneva, 2002), p. 629.

[20] A. Lehrach et al., in Acceleration of Polarized Protons and Deuterons at COSY, edited by Y.I. Makdisi, W.W. MacKay, and A.U. Luccio, AIP Conf. Proc. No. 675 (AIP, Melville, NY, 2003), p. 153.

[21] V. Schwarz et al., in Proceedings of the 13th International High Energy Spin Physics Symposium, Protvino, 1998, edited by N.E. Tyurin et al. (World Scientific, Singapore, 1999), p. 560.

[22] M. Altmeier et al. (EDDA Collaboration), Phys. Rev. Lett. 85, 1819 (2000).

[23] H. Stein et al., At. Energ. 94, 24 (2003).

[24] D. Chiladze et al., Phys. Rev. ST Accel. Beams 9, 050101 (2006).

[25] P. D. Eversheim et al., in The Polarized Ion Source for COSY, edited by E. J. Stephenson and S. E. Vigor, AIP Conf. Proc. No. 339 (AIP, Woodbury, NY, 1995), p. 668.

[26] R. Weidmann et al., Rev. Sci. Instrum. 67, 1357 (1996).

[27] O. Felden et al., in Proceedings of the 9th International Workshop on Polarized Sources and Targets, Nashville, IN, 2001, edited by V. P. Derenchuk and B. von Przewoski (World Scientific, Singapore, 2002), p. 200. 